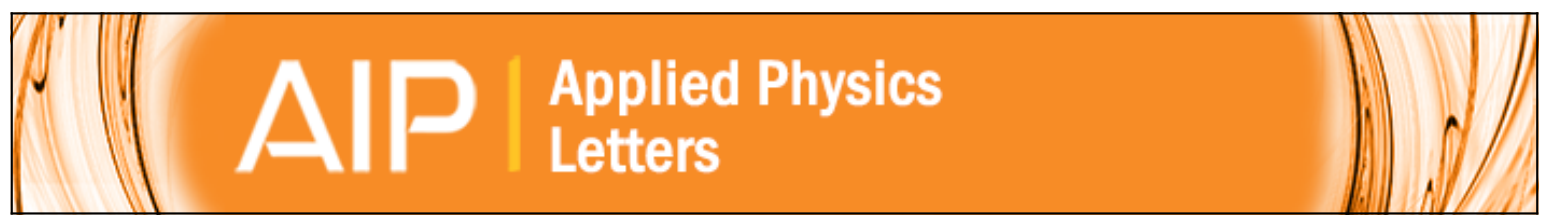

\title{
Extrinsic doping in silicon revisited
}

U. Schwingenschlögl, A. Chroneos, C. Schuster, and R. W. Grimes

Citation: Applied Physics Letters 96, 242107 (2010); doi: 10.1063/1.3455313

View online: http://dx.doi.org/10.1063/1.3455313

View Table of Contents: http://scitation.aip.org/content/aip/journal/apl/96/24?ver=pdfcov

Published by the AIP Publishing 


\title{
Extrinsic doping in silicon revisited
}

\author{
U. Schwingenschlögl, ${ }^{1}$ A. Chroneos, ${ }^{2}$ C. Schuster, ${ }^{3}$ and R. W. Grimes ${ }^{2, a)}$ \\ ${ }^{1}$ PSE Division, KAUST, Thuwal 23955-6900, Saudi Arabia \\ ${ }^{2}$ Department of Materials, Imperial College London, London SW7 2BP, United Kingdom \\ ${ }^{3}$ Institut für Physik, Universität Augsburg, 86135 Augsburg, Germany
}

(Received 24 April 2010; accepted 27 May 2010; published online 17 June 2010)

\begin{abstract}
Both n-type and p-type doping of silicon is at odds with the charge transfer predicted by Pauling electronegativities and can only be reconciled if we no longer regarding dopant species as isolated atoms but rather consider them as clusters consisting of the dopant and its four nearest neighbor silicon atoms. The process that gives rise to n-type and p-type effects is the charge redistribution that occurs between the dopant and its neighbors, as we illustrate here using electronic structure calculations. This view point is able to explain why conventional substitutional $\mathrm{n}$-type doping of carbon has been so difficult. (C) 2010 American Institute of Physics. [doi:10.1063/1.3455313]
\end{abstract}

The standard model for n-type and p-type doping of silicon ( $\mathrm{Si})$ (Refs. 1 and 2) is at odds with the charge transfer predicted by Pauling electronegativities. ${ }^{3}$ Specifically, phosphorous $(\mathrm{P})$ is n-type and is expected to donate an electron to the conduction band but a $\mathrm{P}$ atom is more electronegative than a Si atom-the transfer of charge is therefore toward the P not away from it. Gallium $(\mathrm{Ga})$ is p-type and yet its electronegativity is lower than that of Si.

n-type dopants such as $\mathrm{P}$ have five valence electrons and since only four can participate in forming $s p^{3}$ hybrid bonds with the four neighboring tetrahedral $\mathrm{Si}$, there is a "spare" electron for which there is a low energy for delocalization to the conduction band. If we compare this to the situation that Pauling would describe for molecular $\mathrm{PSi}_{4}$ we find a distinctly different model. The Pauling electronegativity for $\mathrm{P}$ is 2.19 but for $\mathrm{Si}$ it is 1.90; consequently charge is transferred from $\mathrm{Si}$ to $\mathrm{P}$. Of course, it is not necessarily the case that a molecular description is helpful when describing a dopant embedded in a solid but nevertheless, the discrepancy is intriguing. Equivalently, if we consider an example of p-type doping, the electronegativity of Ga is 1.81 so that charge will transfer from $\mathrm{Ga}$ to Si. As a p-type dopant we expect charge to be accumulated by the $\mathrm{Ga}$ atom; again there is an apparent incompatibility.

Since the basic charge conduction mechanisms of $\mathrm{n}$ and p-type $\mathrm{Si}$ are hardly in dispute, we have carried out electronic structure calculations in order to understand the essential charge transfer processes that operate in $\mathrm{P}$ and $\mathrm{Ga}$ doped $\mathrm{Si}$ and thereby offer an explanation, through which the two views can be reconciled.

We use the generalized gradient approximation as implemented in the WIEN2K program package, a full-potential linearized augmented-plane-wave code. ${ }^{4}$ Previous studies have shown that this code is well suitable for describing charge transfer effects, see Ref. 5, for instance. We employed a 2 $\times 2 \times 2 \mathrm{Si}$ supercell unit, which we dope by inserting one impurity atom ( $\mathrm{P}, \mathrm{As}$, or $\mathrm{Ga}$ ) per $64 \mathrm{Si}$ atoms, i.e., we form a high doping level of $1.6 \%$. The impurity-impurity distance is therefore twice the Si lattice constant, $\mathrm{a}_{\mathrm{Si}}=5.43 \AA$. The charge density is represented in our calculations by about 128000 plane waves and the mesh for the k-space integra-

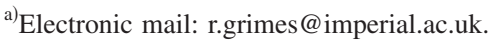

tion consists of 24 points within the irreducible wedge of the Brillouin zone. Specifically to WIEN2K, we set $\mathrm{RK}_{\max }=7$ and choose a muffin tin radius of $\mathrm{R}=2.21$ atomic units for all atoms. The $\mathrm{P} 3 s$; As $3 d, 4 s$; and $\mathrm{Ga} 3 d$ atomic orbitals are treated as semicore states, while the valence states are comprised of the $\mathrm{Si} 3 s, 3 p ; \mathrm{P} 3 p, 4 s ;$ As $4 p, 4 d, 5 s$; and Ga $4 s, 4 p, 4 d$ orbitals.

Figure 1 shows the change in electron density (a difference map) when an electron is removed from an otherwise perfect Si lattice. The map shows a portion of the (110) plane though the Si supercell, which intersects two $s p^{3}$ bonds of a Si tetrahedron. Small amounts of charge have been lost from regions that correspond to the $s p^{3}$ hybridized bonds, evenly throughout the 64 atom supercell (only a section of the whole plane is shown in Fig. 1 and all subsequent figures). This is compatible with the standard model for a hole state at the top of the valence band, that is distributed across the whole cell.

Next, we consider the charge redistribution associated with a P dopant incorporated at a single site in the 64 atom Si

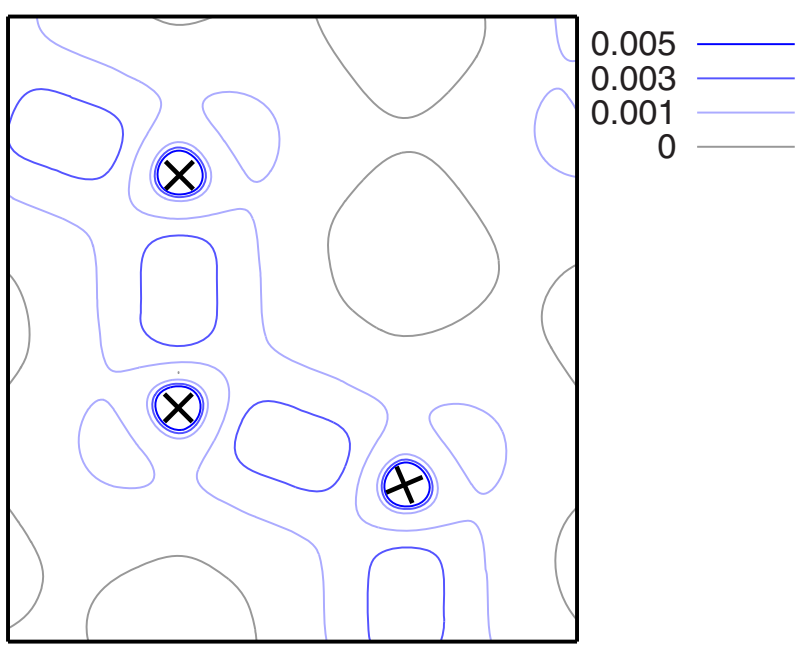

FIG. 1. (Color online) Electron density contour map of the (110) plane through a 64 atom $\mathrm{Si}$ supercell, showing the charge difference between a perfect neutral $\mathrm{Si}_{64}$ supercell and a $\mathrm{Si}_{64}$ supercell with a single positive charge. The negative charge contours indicate where charge has been lost, that is, at the $\mathrm{Si}$ cores and the two $\mathrm{Si}-\mathrm{Si} s p^{3}$ bonds that this section intersects. $\mathbf{X}$ indicates the nucleus of the $\mathrm{Si}$ atoms. 


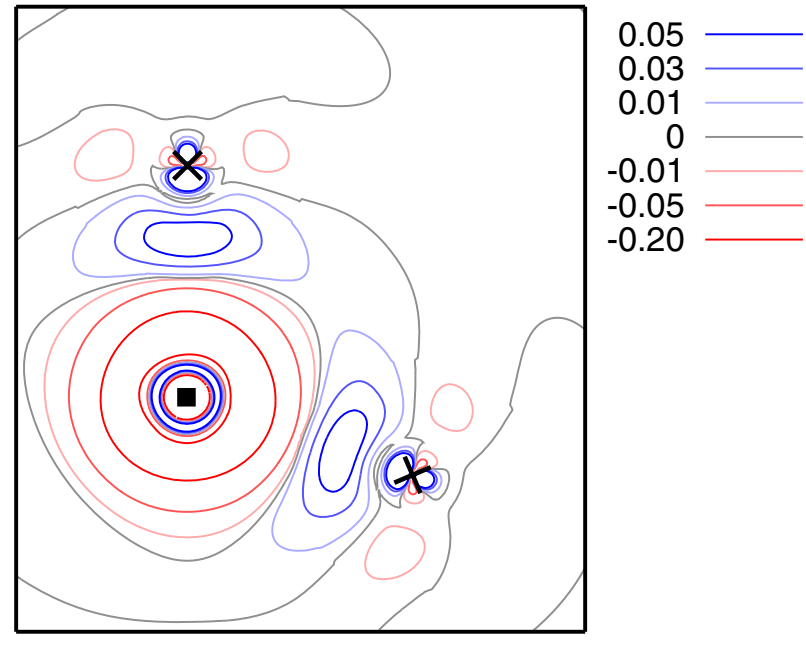

FIG. 2. (Color online) Electron density contour map of the (110) plane that shows the difference between a perfect neutral $\mathrm{Si}_{64}$ supercell and a $\mathrm{PSi}_{63}$ supercell, in the vicinity of the $\mathrm{P}$ dopant. The $\mathrm{P}$ atom accumulates charge and the $\mathrm{Si}$ atoms lose charge from their core regions and from the $s p^{3}$ bonds with the P. $\mathbf{X}$ indicates the position of Si nuclei and $\mathbf{\square}$ indicates the P nucleus.

cell. Figure 2 shows the density difference between the $\mathrm{Si}_{64}$ cell and a $\mathrm{PSi}_{63}$ cell and hence the charge redistribution associated with incorporating the dopant species (focusing on the region around the $\mathrm{P}$ dopant). Of course, this is a $0 \mathrm{~K}$ calculation and as such we do not expect the $\mathrm{P}$ to have donated its electron to the conduction band. The Pauling model for neutral $\mathrm{P}$ doped $\mathrm{Si}$ at $0 \mathrm{~K}$ would predict that charge is donated from the surrounding lattice to the P. This is exactly what is calculated; Fig. 2 shows charge is lost from the neighboring Si atoms, and accumulated in the region of the $\mathrm{P}$ atom. Furthermore, the charge transfer is local, that is, the charge attracted by the $\mathrm{P}$ atom all comes from the four nearest neighbor $\mathrm{Si}$ atoms but not from $\mathrm{Si}$ atoms further into the supercell (beyond the region shown in Fig. 2). Note that the dense positive and negative contours centered at the $\mathrm{P}$ nucleus are due to small changes in position in space of the semicore density maxima and are not due to changes in bonding.

Figure 2 begs the question, what happens in $\mathrm{P}$ doped $\mathrm{Si}$ when an electron is removed; where is it removed from? The standard model suggests it is entirely lost from the $\mathrm{P}$ dopant-which one might particularly expect, given the charge transfer to P. Figure 3 shows the change in charge density when an electron is removed from a $\mathrm{PSi}_{63}$ cell (i.e., the electron density difference between neutral $\mathrm{PSi}_{63}$ and $\mathrm{PSi}_{63}{ }^{+}$). Remarkably, it is found that charge is lost not only from the $\mathrm{P}$ but also the nearest neighbor $\mathrm{Si}$ atoms, despite these lattice species having already been depleted of some charge. So, what is the magnitude of the charge lost by the $\mathrm{P}$ in comparison to the charge it accumulated from the nearest neighbor Si atoms? This is illustrated by Fig. 4, which shows the difference between a perfect $\mathrm{Si}_{64}$ cell and a $\mathrm{PSi}_{63}{ }^{+}$cell. It is at once apparent that, contrary to the idea of the $\mathrm{P}$ has donated charge, the missing electron has come entirely from the four near neighbor $\mathrm{Si}$ atoms. In fact, the $\mathrm{P}$ atom has still accumulated charge, that is, it is still negatively charged, albeit not as greatly as it was in the neutral lattice (compare Figs. 3 and 4). Calculations for As doping (Pauling electronegativity 2.18 ) revealed equivalent results. We therefore

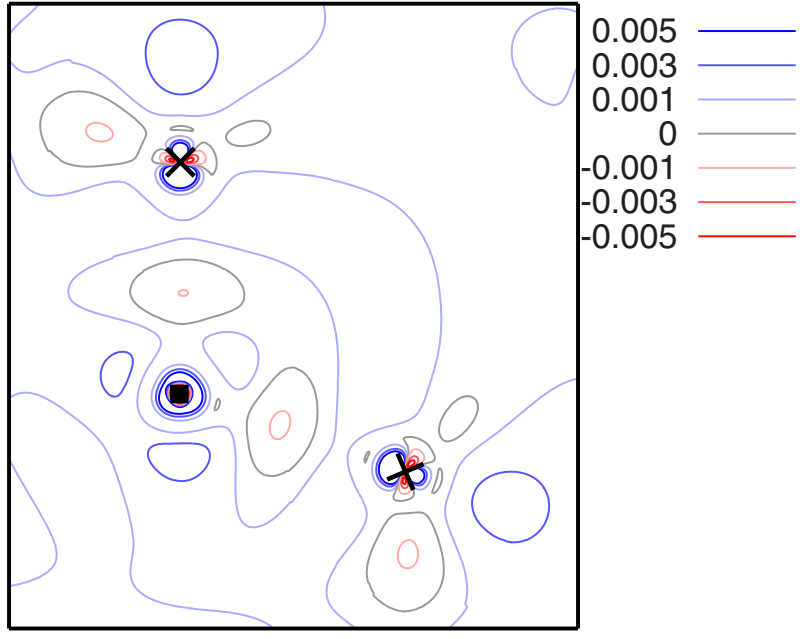

FIG. 3. (Color online) Electron density contour map of the (110) plane that shows the difference between a neutral $\mathrm{PSi}_{63}$ supercell and a positively charged $\mathrm{PSi}_{63}{ }^{+}$supercell. The negative charge contours indicate where charge has been lost, that is, from both $\mathrm{P}$ and $\mathrm{Si}$ atoms. $\mathbf{X}$ indicates the position of Si nuclei and $\mathbf{\square}$ indicates the $\mathrm{P}$ nucleus.

suggest that a more appropriate model for n-type doping is the promotion of an electron from a cluster consisting of four $\mathrm{Si}$ atoms, surrounding a dopant species that has an electronegativity greater than that of the host lattice. Our assumption here is that the promoted electron, since it is delocalized over the whole lattice, will not affect the localized hole state and as such the $\mathrm{PSi}_{63}{ }^{+}$supercell provides a reliable model.

Turning now to p-type dopants, Fig. 5, which reports the difference between a $\mathrm{Si}_{64}$ atom cell and a $\mathrm{GaSi}_{63}$ cell, illustrates charge donation from the less electronegative $\mathrm{Ga}$ (Pauling electronegativity 1.81) to the nearest neighbor $\mathrm{Si}$ atoms. Again the Pauling model is supported by the calculations. Figure 6 is then the difference map between a neutral $\mathrm{GaSi}_{64}$ supercell and a $\mathrm{GaSi}_{63}{ }^{-}$supercell that has assumed an additional single charge. It shows that the additional electron is not localized only on the $\mathrm{Ga}$ atom but trapped by the $\mathrm{GaSi}_{4}$ cluster. The assumption we have made in deriving this model for p-type behavior is that the delocalized hole state at the

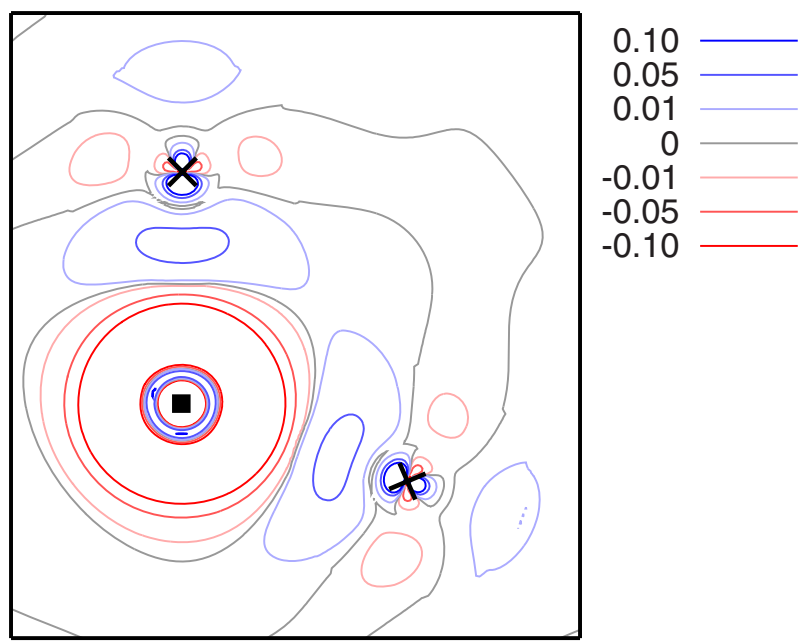

FIG. 4. (Color online) Electron density contour map of the (110) plane that shows the difference between a perfect neutral $\mathrm{Si}_{64}$ supercell and a positively charged $\mathrm{PSi}_{63}{ }^{+}$supercell. The contours indicate that charge has been lost only from the $\mathrm{Si}$ atoms, while the $\mathrm{P}$ atom has accumulated charge. $\mathbf{X}$ indicates the position of Sinvclei and $\square$ indicates the $P$ nucleus 


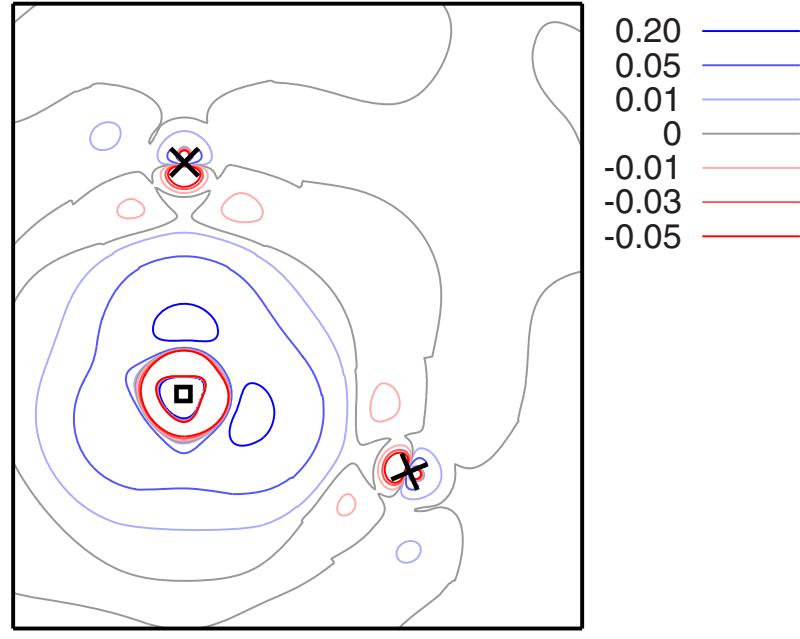

FIG. 5. (Color online) Electron density contour map of the (110) plane that shows the difference between a perfect neutral $\mathrm{Si}_{64}$ supercell and a $\mathrm{GaSi}_{63}$ supercell, in the vicinity of the Ga dopant. The Ga atom donates charge to the Si atoms. $\mathbf{X}$ indicates the position of Si nuclei and $\diamond$ indicates the Ga nucleus.

top of the valence band (as in Fig. 1) will not significantly influence this local cluster based electron trap state.

Finally we consider the difficulties experienced with attempts to substitutionally dope diamond to form shallow donors. ${ }^{6,7}$ If diamond were to follow the same pattern as $\mathrm{Si}$, to achieve a useful n-type material it would be a necessary (but probably not sufficient) condition to dope with an element that has an electronegativity greater than carbon. Unfortunately, the standard n-dopant elements, $\mathrm{P}, \mathrm{As}$, and $\mathrm{Sb}$ all have electronegativities $(2.19,2.18$, and 2.05 , respectively), which are smaller than that of carbon (value 2.55). Con-

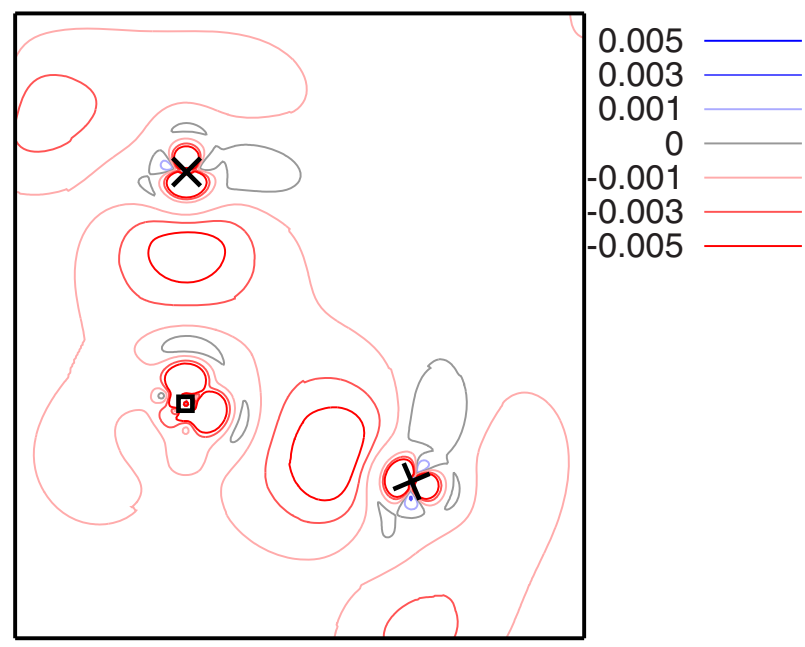

FIG. 6. (Color online) Electron density contour map of the (110) plane that shows the difference between a perfect neutral $\mathrm{Si}_{64}$ supercell and a negatively charged $\mathrm{GaSi}_{63}{ }^{-}$supercell. The contours indicate that charge has been gained only from $\mathrm{Si}$ atoms, while the $\mathrm{Ga}$ atom has lost charge. $\mathbf{X}$ indicates the position of Si nuclei and $\square$ indicates the P nucleus. versely, on the same basis, p-type diamond would be expected since conventional p-type dopants have smaller electronegativities than carbon. Clearly, a simple observation as presented here, can only offer a simple indication, while there is a rich detail for each dopant presently being investigated by others (see, for example, Refs. 8 and 9).

Electronic structure calculations have revealed that, contrary to the model that n-type dopants donate charge when an electron is promoted, it is the four surrounding $\mathrm{Si}$ atoms which provide the charge, at least with respect to the defect free perfect lattice. This observation can be understood if we consider that when an n-type dopant such as $\mathrm{P}$ or As is substituted into the $\mathrm{Si}$ lattice, it forms a cluster with four $\mathrm{Si}$ atoms since only those neighbors donate charge to the more electronegative dopant atom. When a single charge is removed from a supercell containing this cluster it is lost, for example, from the $\mathrm{PSi}_{4}$ cluster to form a local hole state. As such, it is the $\mathrm{PSi}_{4}$ cluster that is the vehicle for n-type doping. Equivalently, in p-doped $\mathrm{Si}$, it is the $\mathrm{GaSi}_{4}$ cluster that is able to attract an electron rather than the $\mathrm{Ga}$ atom itself. Thus, the role of the dopant ions is to establish a suitable local environment that delivers the n-type or p-type behavior. Such a cluster based model establishes the impetus to consider a much wider selection of potential dopant species (i.e., types of clusters) as is already underway (see, for example, Ref. 9). It also provides a rational for why n-doped diamond has been difficult to achieve and may aid in our understanding of defect mediated deactivation processes in heavily doped and/or alloy semiconductors. ${ }^{10-15}$

This publication was based on work supported in part by King Abdullah University of Science and Technology.

${ }^{1}$ C. Kittel, Introduction to Solid State Physics, 7th ed. (Wiley, New York, 1996), Chap. 8, p. 221.

${ }^{2}$ N. W. Ashcroft and N. D. Mermin, Solid State Physics (Harcourt, Fort Worth, 1976), Chap. 28, p. 577.

${ }^{3}$ L. Pauling, J. Am. Chem. Soc. 54, 3570 (1932).

${ }^{4}$ P. Blaha, K. Schwarz, G. Madsen, D. Kvasicka, and J. Luitz, Wien2k: An Augmented Plane Wave Plus Local Orbitals Program for Calculating Crystal Properties (Vienna University of Technology, Vienna, 2001).

${ }^{5}$ U. Schwingenschlögl and C. Schuster, Phys. Rev. Lett. 102, 227002 (2009)

${ }^{6}$ M. A. Pinault, Physica B 401-402, 51 (2007).

${ }^{7}$ M. Nesladek, Semicond. Sci. Technol. 20, R19 (2005).

${ }^{8}$ B. Butorac and A. Mainwood, Phys. Rev. B 78, 235204 (2008).

${ }^{9}$ D. Segev and S. H. Wei, Phys. Rev. Lett. 91, 126406 (2003).

${ }^{10} \mathrm{~A}$. Chroneos, C. Jiang, R. W. Grimes, U. Schwingenschlögl, and H. Bracht, Appl. Phys. Lett. 95, 112101 (2009).

${ }^{11}$ S. Brotzmann, H. Bracht, J. Lundsgaard Hansen, A. Nylandsted Larsen, E. Simoen, E. E. Haller, J. S. Christensen, and P. Werner, Phys. Rev. B 77, 235207 (2008).

${ }^{12}$ A. Chroneos, R. W. Grimes, and H. Bracht, J. Appl. Phys. 106, 063707 (2009).

${ }^{13}$ G. M. Lopez, V. Fiorentini, G. Impellizzeri, S. Mirabella, and E. Napolitani, Phys. Rev. B 72, 045219 (2005).

${ }^{14}$ A. Chroneos, H. Bracht, R. W. Grimes, and B. P. Uberuaga, Appl. Phys. Lett. 92, 172103 (2008).

${ }^{15}$ A. Chroneos, R. Kube, H. Bracht, R. W.Grimes, and U. Schwingenschlogl, Chem. Phys. Lett. 490, 38 (2010). 